\title{
Improving Users' Comprehension of Changes with Animation and Sound: An Empirical Assessment
}

\author{
Céline Schlienger ${ }^{1}$, Stéphane Conversy ${ }^{2,3}$, Stéphane Chatty ${ }^{1,2}$, Magali Anquetil ${ }^{1}$, \\ and Christophe Mertz ${ }^{1}$ \\ ${ }^{1}$ IntuiLab \\ Les Triades A - Rue Galilée - BP 77242, 31672 Labège Cedex \\ \{celine, chatty, anquetil, mertz\}@intuilab.com \\ ${ }^{2}$ ENAC - Ecole Nationale de l'Aviation Civile - ${ }^{3}$ DSNA/DTI-R\&D \\ 7, avenue Edouard Belin, 31055 Toulouse \\ stephane.conversy@enac.fr
}

\begin{abstract}
Animation or sound is often used in user interfaces as an attempt to improve users' perception and comprehension of evolving situations and support them in decision-making. However, empirical data establishing their real effectiveness on the comprehension of changes are still lacking. We have carried out an experiment using four combinations of visual and auditory feedback in a split attention task. The results not only confirm that such feedback improves the perception of changes, but they also demonstrate that animation and sound used alone or combined bring major improvements on the comprehension of a changing situation. Based on these results, we propose design guidelines about the most efficient combinations to be used in user interfaces.
\end{abstract}

Keywords: Empirical evaluation, animation, sound, multimodal feedback, comprehension of changes, dual task.

\section{Introduction}

Animation or sound are often suggested for improving feedback on users' actions, for notifying changes, or for improving awareness of more complex but although frequent situations where users need to split their attention between multiple information sources. User Interface designers often have assumptions about the effectiveness of animation or sound as feedback to monitor an evolving situation (see [1] and [18] for examples). In this work, we address the question of the real effectiveness of animation, of sound and of the combination of both modalities in situations where changes occur. We propose ways to use each modality in such situations, and provide experimental evaluations of their benefits, be they used alone or combined.

In order to demonstrate the efficiency of sound and animation, we used the Situation Awareness framework proposed by Endsley [8]. Situation Awareness (SA) is defined by Ensley as "knowing what is going on around you", so that one can react appropriately to situations. Endsley identifies three increasing levels of situation awareness: (1) the perception of changes in the environment, (2) the comprehension 
of their meanings, and (3) the projection of their status in the near future [8]. Our study concerns the two first levels, with a particular focus on the second one: if empirical data on the effectiveness of animation or sound on the perception of changes do exist, empirical data establishing their effectiveness at helping users to understand changes are still lacking.

After reviewing previous work on the use of animation and sound in user interfaces, we explain our use of the modalities and our experimental model. We then describe the experiment and analyze its quantitative results. Finally, we discuss the interpretation and consequences of these results.
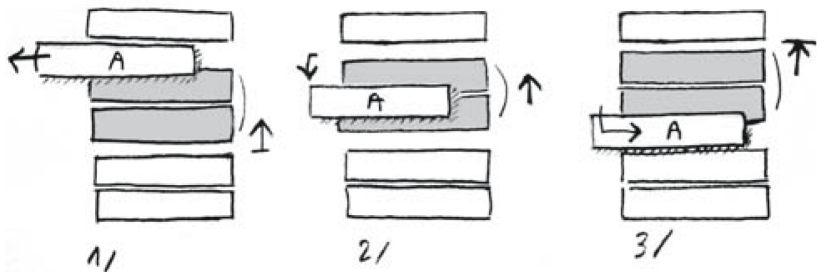

Fig. 1. Storyboard of the animation: changing element (A) moves in parallel with in-between elements (colored in grey)

\section{Related Work}

This section presents empirical studies or major uses of animation or sound in user interfaces, as well as work on their combination with other modalities. We classify them according to the two first levels of Situation Awareness: perception and comprehension.

\subsection{Enhancing Perception}

The first level of situation awareness is the perception of changes. Motion is particularly suited to attract the user's attention, because it is a pre-attentive visual feature: its detection happens at the early stages of visual perception [22,25]. Athènes et al. found that motion has a statistically significant positive impact on the time the user requires to detect alarms [1]. Bartram et al. experimentally established that motions in peripheral vision are particularly effective at grabbing attention, especially compared to other animated graphical attributes, such as shape or color [2].

Sound brings information regardless of the user's focus of attention. Thus, a large body of works showed that the audio channel is a very appropriate modality for notification of changes (see $[17,24]$ for examples). Combined with graphics, sound is also supposed to support users in direct manipulation interaction by enhancing the perception of their actions $[6,13,20]$.

\subsection{Enhancing Comprehension}

The second level of situation awareness is the comprehension of the meaning of changes, i.e. the "integration of multiple pieces of information and the user's determination of their relevance on his/her goals" [8]. 
Sound has been shown to help users monitor background processes [7]. For example, in the Sonic Finder [9], the sound of pouring water accompanies a graphical progress bar during long processes i.e. copying a large amount of files. The use of the audio channel enables a user to engage in another activity while monitoring the progression of the background process. Though not formally assessed, the Arkola simulation illustrates how sound conveys information about the ongoing activity of remote participants, so as to help the user to understand the evolution of a collaborative task [10].

Animation has been experimentally shown to be effective at replaying missed changes [4] and to support the comprehension of textual information displayed in the periphery [14]. Many other works hint at the effectiveness of animation as feedback, but real effectiveness has not been experimentally established yet. Feedback with animation is supposed to help users understand the consequence of their actions and the cause of a change [5,15,21]. Animation as feedback might also be effective at supporting collaborative work, when co-workers must be aware of what is happening on neighbors' screen [15]. Animation is also used to support users in keeping track of the relationships between changing graphical elements in complex visualizations $[12,18]$, but no experimental study proves its effectiveness. Our work aims at assessing the benefits of using animation and sound for this particular use: supporting changes comprehension.

\section{The Experiment}

In this work, we study the impact of animation and sound, used alone or combined, on the perception and the comprehension of situations where changes occur. We chose to study situations where the user shares attention between distant sources from which he/she has to gather up-to-date information to perform a task, such as monitoring incoming mail while editing a spreadsheet. Such situations are good candidates for improvement with animation feedback and sound feedback. First, changes can occur outside the user's focus of attention and can be missed if the interface makes use of static graphics only. The notification effect of animation and sound may improve the user's perception of the changes. Second, sound and animation can be used to extract the relevant data of the change and they may help identifying their value.

Our study relies on a simulation of an Air Traffic Control task. Controllers in an airport tower manage aircraft, busses and other vehicles moving on the ground. They schedule concurrent access on runways and taxiways. They split their attention between actual traffic observed through the window and the traffic previsions displayed on monitoring screens; they then give orders to drivers and pilots on the radio. We designed an interface that shows in real-time the planned schedule of mobile objects at a particular location on the ground, say a crossroad between a runway and a taxiway. To simulate situations where a user splits attention between distant sources, the experiment followed a dual task paradigm: while performing a demanding main task designed to focus attention, subjects must keep track of changes occurring in the list displayed in their peripheral vision (see Fig. 2). 


\subsection{Experimental Model}

All changes in a computing environment consist of data variations. As observed by Bertin, visual representations are mappings of that data to graphical dimensions, such as color, shape, or position [3]. When a piece of data changes, its visual representation changes as well. When the change is smooth, a user can catch it: an animation is a smooth change of a visual dimension, be it position, color, or shape.

We chose a list of ranked items as the object to monitor in the experiment. A list is a good candidate to model changing situations with animation: as rank is coded with position, a change in rank leads to a change of position, which can be reinforced by a smooth motion from the origin to the destination. Furthermore, a number of user interfaces use dynamic, ordered lists to display information: names of participants in a chat session, drivers' rank in racing car video games, or CPU consumption of processes in some utilities.

When a change occurs, the evaluation of invariant parameters associated with the changing item is essential to understand the new situation. Thus, the list is composed of elements of different types that do not change, but that a user has to recognize when a change of position occurs. Types are coded with a graphical icon and with a sound, as sound is often used to convey type through a metaphor [9].

We measure a user's situation awareness at the first two levels of situation awareness by quantifying perception as a user's ability to detect changes occurring in the list, and comprehension as a user's ability to correctly identify static and dynamic parameters of the list. The parameters are: the type of the object involved in the change, the rank in the list of the object before the change and the rank in the list of the object after the change.

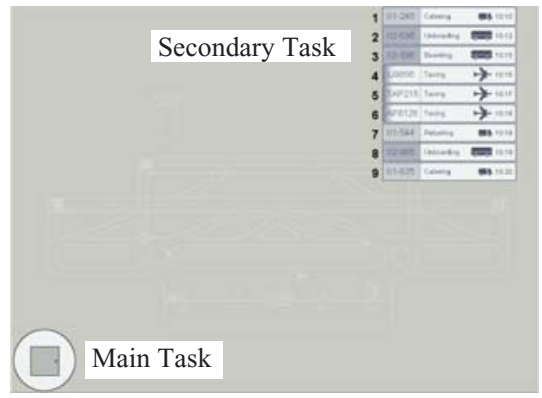

(a)

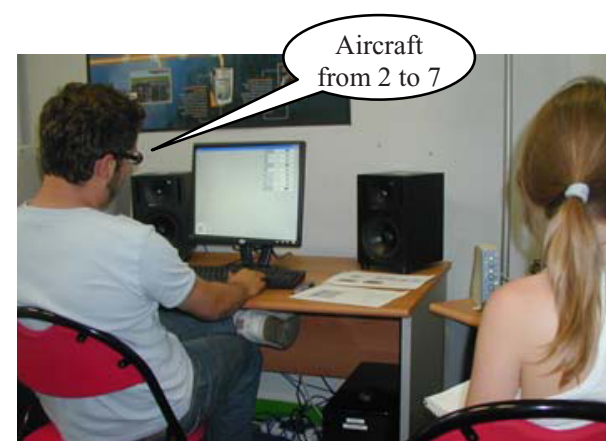

(b)

Fig. 2. (a) The experiment interface. (b) The experimenter (on the right) records on a dedicated interface the subjects (on the left) answers.

\subsection{List Design}

The list contains nine rectangular elements: three aircraft, three busses and three vehicles (Fig. 3). Each element includes four graphical attributes: a unique identifier in a colored zone, the status of the mobile object, an icon, and a time of transit on the crossroad. The identifier, the status and the icon are specific to the 
object type (aircraft, bus or vehicle). The list is sorted vertically according to the reference time. Users can read the rank of an item with a static digit from 1 to 9 located on the left of the item (Fig. 2a). When the estimated time of transit of an element changes (for example, because a flight is delayed), its new position is computed and the list is updated. In the simulation, elements never disappear from the list, nor do they appear: the time of transit is never reached, and no new mobile object is scheduled.

\subsection{Tasks and Setting}

Main task. The main task is a perceptive identification task. A point appears at random positions in the set (top, bottom, left, right) inside a static square. The pace of appearance is randomly chosen, with an average of 1.1 changes per second. Subjects are asked to identify the position of the point and to press the matching key of the keyboard (up, down, left or right). If an error occurs, a circle displayed around the square is colored in red. Subjects were asked to focus their attention on this main task with the no-error objective.

Secondary task. The secondary task consists of perception and comprehension of the changes occurring in the list. When perceiving a change, the subject was asked to say the type of the moving element, its origin position and its destination position. Should he/she not be able to evaluate all three parameters, the subject would enumerate those recognized or say, at least, that something has moved. The experimenter used a dedicated interface to record subjects' answers (see Fig. 2b). Changes occurred at random time, with an average period of 10 second between two changes.

Setting. The square of the main task is displayed at the bottom left of a 19' screen, $25 \mathrm{~cm}$ away from the list displayed at the top right (see Fig. 2a). The user sits $80 \mathrm{~cm}$ away from the screen. There is a 17 degrees vision angle between the two zones, guaranteeing peripheral vision conditions.

\subsection{Experimental Conditions}

We tested the following four combinations of visual and auditory feedback (Fig. 3).

No animation - no sound. This is the control condition. When the rank of an item changes, the graphical scene is instantaneously swapped for an updated one.

No animation - sound. Before the graphical scene swaps, a sound specific to the object type is played. The sounds we designed are metaphorical, and code the object type (aircraft, bus, and vehicle). They are played through two speakers, but they are not spatialized. The type of sound is the only auditory variable.

Animation - no sound. The element smoothly moves from its origin position to its destination position. The animation involves two motions occurring in parallel as illustrated in Fig. 1: 
a) a motion of the element concerned by the change in three steps:

- move to the left of the list by $20 \%$ of the element width, during $300 \mathrm{~ms}$,

- move down or up to destination ordinate during a time computed as: $400 \mathrm{~ms}+$ abs(destination position - origin position)*100 ms,

- move to the right to destination position during $300 \mathrm{~ms}$.

b) a motion of the set of elements between origin and destination position: the origin position left blank is smoothly filled by the elements to empty the destination position.

Animation - sound. Before the element moves in the list, the sound specific to the type is played. Then, the element smoothly moves from its original position to its destination position (Fig. 1). The sounds used in this condition are the same as those used in the no animation - sound condition.

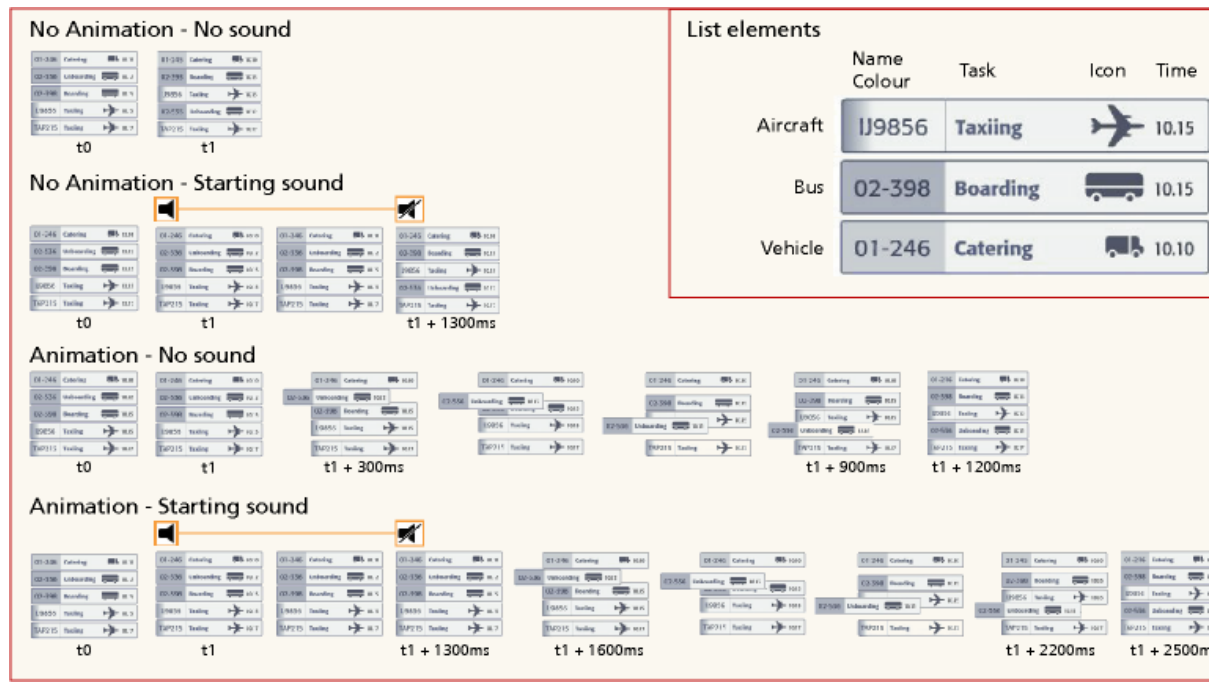

Fig. 3. Experimental conditions and composition of the list elements

\subsection{Experiment Design}

To get reference performances, the main task was first evaluated without the secondary task. This condition enables to evaluate the degradation of the main task performances by the introduction of the secondary task with each feedback. Then, all subjects performed main and secondary tasks under all conditions. The conditions were passed randomly to avoid order and learning effects. For each condition, three changes per object type occurred randomly in the list (to avoid order effect too); thus, there were 3 changes involving aircraft, 3 involving busses and 3 involving vehicles.

Each condition began with a learning phase during which the user performed the secondary task only. In particular, this phase allowed the user to become familiar with 
the animation and sound. The learning phase stopped when the user was able to perform the task with no errors (except in no animation - no sound condition, where it is very difficult to get the correct answer even with attention focused on the list).

All subjects were given a post-experiment questionnaire aimed at collecting their opinion about the impact of animation and sound on their situation awareness.

24 subjects volunteered for the experiment ( 8 females, 16 males). For results to be generic, we chose subjects in different activity domains (HCI research, healthcare, commerce, administration, air traffic control, art, culture, education, computer science) and from various ages (from 24 to 45 with an average age of 32 ).

\subsection{Hypotheses}

From the literature, we know that animation or sound is efficient at grabbing the user's attention in peripheral vision. What we tested in this experiment is whether animation and/or sound are able improve the comprehension of the changes. Hence, the following hypotheses were formulated:

- Animation and/or sound improve change detection. (HI)

- Animation improves object type identification. (H2)

- Sound improves object type identification. (H3)

- Animation improves origin position identification. (H4)

- Animation and sound improves origin position identification. (H5)

- Animation improves destination position identification. (H6)

\section{Results}

The normality of the gathered data was tested using the Shapiro-Wilk's test. The result shows that the distribution of the data is skewed, so we performed nonparametric tests. For matched samples, the appropriated tests are: 1) the Friedman test that enables to compare all conditions and identify if there are significant results within the data, and 2) if the Friedman test is significant, the Wilcoxon pair-wise test that enables to compare two conditions.

\subsection{Main Task}

The number of errors in the main task is significantly higher when the secondary task is introduced ( $\mathrm{p}<0.001$, Friedman). This result was anticipated, as main and secondary tasks are parallel tasks that require split attention. With the secondary task, pair-wise tests show that the number of errors in the main task is not significantly different between the conditions, except between the no animation - no sound and the animation - starting sound conditions. Hence, for all but one pair, we can attribute the significant improvement on the secondary task reported in the next sections to the difference of feedback, as expected.

Concerning the no animation - no sound and animation - sound conditions, pairwise tests show a slight but significant $(\mathrm{p}=0.04744)$ decrease in performance in the main task ( $1.5 \%$ more errors for animation-sound condition). Users might have split slightly differently their attention resources between main and secondary task in these 
two conditions, precluding any firm conclusion about the effect of feedback in the secondary task. However, the large increase of performance in the secondary task (an average of $75 \%$ fewer errors for animation-sound condition) hints at a significant contribution of feedback. Further testing is needed to evaluate the real effect of feedback between these two particular conditions.

\subsection{Detection}

There is a significant effect of the feedback on list change detection $(p<0.001$, Friedman): while $20 \%$ of changes were missed with no feedback, no change was missed with animation and/or sound. Pair-wise tests show a significant difference $(\mathrm{p}<0.001)$ of no animation - no sound condition ( $80 \%$ of detections) in comparison to the other conditions (100\% of detections). This result confirms hypothesis H1: animation and/or sound improve list changes detection.

\subsection{Object Type Identification}

We wanted to evaluate the subject's comprehension of the situation once he/she has detected a change. Thus, the percentages of identification of the object type, and those of the origin and the destination position presented in the following sections, are calculated on the basis of detected objects.

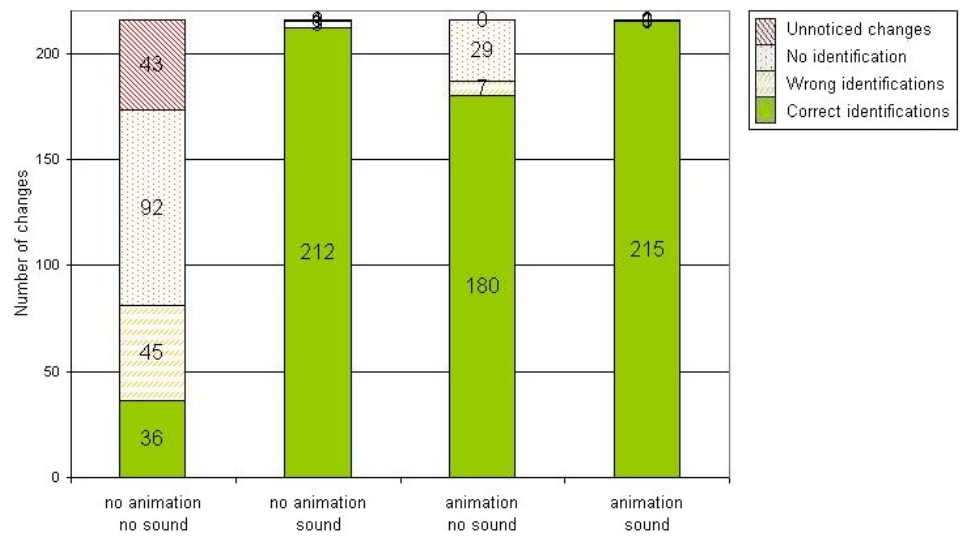

Fig. 4. Results for object type identification

There is a significant effect of the feedback on the object type identification $(\mathrm{p}<0.001$, Friedman). Fig. 4 shows a very high number of correct identifications with animation and almost no errors with sound.

Pair-wise tests show there is a significant difference between no animation - no sound and animation - no sound with respectively $21 \%$ and $83 \%$ of correct answers $(\mathrm{p}<0.001)$. This result confirms hypothesis $\mathrm{H} 2$ : animation improves object type identification.

The two conditions with sound provide more than $98 \%$ of correct answers with no significant difference between them. There is a significant difference between: 
- no animation - no sound and no animation - sound $(\mathrm{p}<0.001)$,

- animation - no sound and animation - sound $(\mathrm{p}<0.001)$

These results confirm hypothesis $H 3$ : sound improves object type identification.

\subsection{Origin Position Identification}

There is a significant effect of the feedback on the identification of the origin position of the object in the list $(\mathrm{p}<0.001$, Friedman). Fig. 5 shows a high number of correct identifications with animation and an even higher when animation is combined with sound.

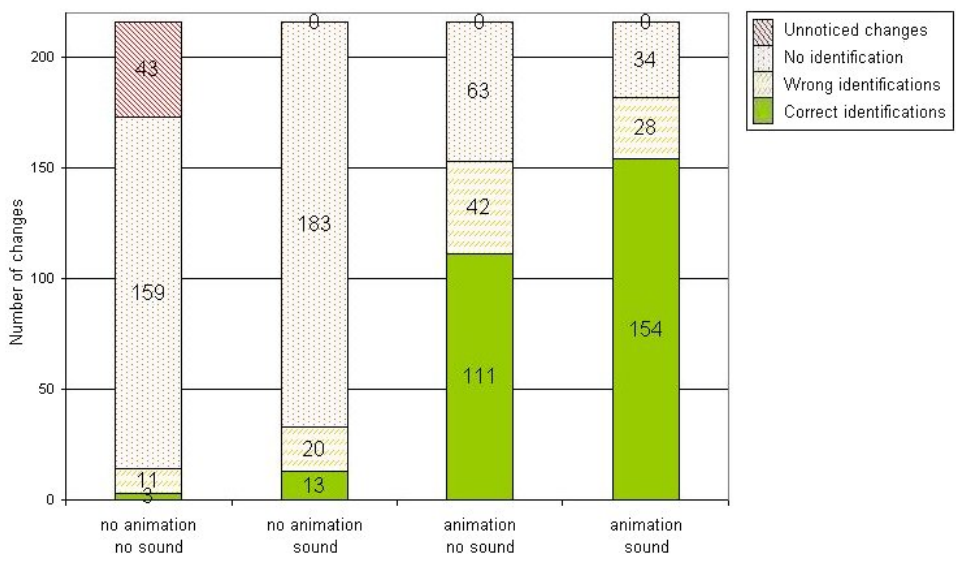

Fig. 5. Results for origin position identification

Pair-wise tests show significant difference between no animation - no sound and animation - no sound with respectively $1.5 \%$ and $51 \%$ of correct answers $(\mathrm{p}<0.001)$. This result confirms hypothesis $H 4$ : animation improves origin position identification in comparison to no animation.

With no animation, there is no significant effect of sound on origin position identification. With animation, there is a significant effect of sound on origin position identification $(\mathrm{p}<0.05)$. This result confirms hypothesis $H 5$ : animation and sound improves origin position identification in comparison to animation.

\subsection{Destination Position Identification}

There is a significant effect of the feedback on the identification of the destination position ( $p<0.001$, Friedman). Fig. 6 shows an increase of correct identifications with animation and/or sound; the number of correct identifications is very high with animation, and even higher when animation is combined with sound.

Pair-wise tests show there is a significant difference between no animation - no sound and animation - no sound with respectively $7.5 \%$ and $78 \%$ of correct answers $(\mathrm{p}<0.001)$. This result confirms hypothesis $H 6$ in that animation improves destination position identification in comparison to no animation. 


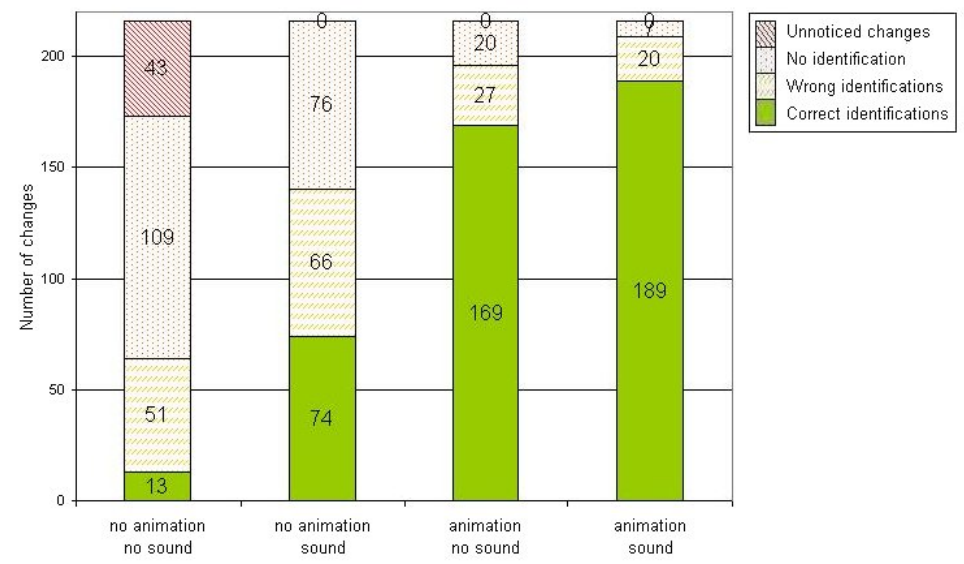

Fig. 6. Results for destination position identification

With no animation, sound has a significant effect on destination position identification (Correct answers: no animation - no sound: $7.5 \%$; no animation sound: $34 \%$; $<<0.001)$. This result shows that without animation, sound significantly improves destination position identification in comparison to no sound.

With animation, there is no significant effect of sound on destination position identification.

\section{Discussion and Implications for Design}

The results of the experiment show that animation and sound, used alone and combined, have effective benefits on awareness of situations where changes occur. Animation and sound improve the perception (first level of situation awareness) of changes and the comprehension (second level) of the evolving situation as the user correctly identifies data relevant to the change. In this section, we translate our results into practical design guidelines dedicated to user interface designers so that they can efficiently use animation and sound to improve users' awareness of a changing situation.

\subsection{Notify Changes with Animation and/or Sound}

Animation and/or sound can be used very efficiently to notify changes occurring on graphic data displayed in peripheral vision. Alone and combined, they enable to perceive all changes, bringing major improvements on the first level of situation awareness. Our results on animation used alone corroborate the results on motion that Bartram provided [2], when she found that less than $1 \%$ of changes were missed in peripheral vision. Usually, sound is used to notify changes that happen outside of the user's vision. Our experiment shows that sound also has a real positive impact as an aid for the detection of changes occurring on visual objects that are displayed in peripheral vision. 
The combination of animation and sound is at least as efficient as each modality used alone to notify changes. Since the use of animation and sound alone yields a $100 \%$ detection rate, we were not able to say if their combination improves performance. In additon, this gives potential solutions to change-blindness [19]. Actually, the combination offers a major advantage: the redundancy of visual and auditory information. If one modality is overloaded or degraded, the other persists and can ensure detection.

\subsection{Maximize the Identification of Essential Static Data with Sound}

When a change occurs, identifying static data might be essential for the user to understand the new situation. Various graphical dimensions are offered to user interface designers to code such data: texts, colors, icons, etc. Our experiment shows that motion significantly improves the identification of data coded with graphics (the object type) when the object position changes (21\% vs. 83\%). Most subjects commented: "with animation, it is easier [than without] to catch the object concerned by the change, and then to look at the icon to identify the object type". However, some of them had difficulties to recognize it while the object moves and their attention is monopolized by the main task.

Auditory feedback outclasses visual feedback with more than $98 \%$ of recognition of the object type. This result provides confirmation of the assumption on redundancy made by Gaver in the SonicFinder [9], where both the icon and the sound encoded the "file" or "directory" type. In contrast to animation, the identification of the data coded by sound is not weakened when the graphical object moves.

Thus, when a change occurs, if a data displayed with graphics is essential to capture, animation can be efficiently used to improve the identification of its value. But user interface designers should prefer sound if they want to optimize identification and provide the best improvements on the second level of SA.

\subsection{Improve the Evaluation of Dynamic Data with Motion}

Values of a dynamic data can be coded by positions of a graphical object. When a change occurs on the data, the position of the object changes. Our experiment shows that the identification of the origin and destination positions of the object can be very efficiently improved with motion, improving thus the evaluation of the data.

The good results of positions identification can be explained by the notification effect of animation: the user look at the object when it smoothly moves from origin to destination position, but not only. As shown in Fig. 1, the animation is composed of two motions: one motion for the element whose rank changed, and one for the set of elements that glide between the origin and destination positions. As the empty space left by the changing element is progressively filled, the user has the whole duration of the animation to catch the old position before it completely disappears. In the same way, the empty space left by the set of in-between elements progressively appears, and enables the user to see in advance the destination of the changing element, before it reaches it. This design can be contrasted to one of a speed gauge, in which a user can perceive a motion, but not the starting nor the ending points. 
This result is a clear improvement over Bartram's findings on benefits of animation [2]: the experiment not only shows that users are able to detect and identify changing items, but also that they can evaluate parameters of the change thanks to the animation. As such, it is an assessment of the assumption that animation may help users understand what is happening, as assumed in [18].

\subsection{Optimize the Comprehension of a Changing Situation by Combining Animation and Sound}

The experimental data enabled us to rank the tested combinations of visual feedback and auditory feedback. Table 1 presents a synthetic view of the results. Each combination gets a score according to users' results. They are evaluated from 1 (best) to 4 (worst). If there is no significant difference between two results, they get the same score. They are then ordered according to these marks. It is interesting to note that subjects, who were asked to rank the feedback by preference order after the experiment, provided the same results. They were not disturbed by any of those, found them useful and understood the benefits they provide.

Table 1. Ranking of visual and auditory feedback

\begin{tabular}{|l|c|c|c|}
\cline { 2 - 4 } \multicolumn{1}{c|}{} & Object attribute & Origin position & Destination position \\
\hline 1. Animation - Sound & $\mathbf{1}$ & $\mathbf{1}$ & $\mathbf{1}$ \\
\hline 2. Animation - No sound & 3 & 2 & 1 \\
\hline 3. No animation - Sound & 1 & 3 & 3 \\
\hline 4. No animation - No sound & 4 & 4 & 4 \\
\hline
\end{tabular}

Table 1 shows that the combination of animation and sound provides the best comprehension of the changing situation: it provides the bests results in the identification of the essential static data and in the evaluation of the changing parameter. The improvement provided by the addition of sound to animation can be first explained by the notification effect of the sound: it notifies a change about to happen; the user focuses his/her attention on the list just before it changes and sees the animation. In addition, combining auditory and visual feedback offers the opportunity to specialize attention channels [23]. Auditory feedback can be used to identify the object type while visual feedback can be used to recognize the positions. This strategy was reported by subjects themselves and confirmed by the experimenter who noticed that subjects give the object type before the animation begins. Further studies should be conducted to evaluate each effect: notification effect and attention channels specialization.

\section{Conclusion}

In this study, we addressed the problem of the comprehension of changes when the attention of a user is split between different information sources. We have conducted 
an experiment that tested combinations of graphical, animated and auditory feedback to improve detection and evaluation of changes occurring in a list displayed in peripheral vision. We found that the use of animation and sound enables users to notice all changes, in conformance with previously available results. In addition, we found that sound can reinforce invariant data coded with graphics such as the type of an element. We also found that motion helps in identifying the old and new values of changing positions, and that a sequential combination of sound and animation is the most efficient feedback for conveying information to users. These results lead us to conclude that the use of animation and sound, alone and combined, improves users' situation awareness, for the perception and comprehension levels.

Stakeholders of interactive systems projects are often concerned with the fact that few works show that rich interfaces are actually more effective than classic ones, and question the extra expense needed for their design. Our results show statistically significant advantages when using animation and sound feedback in user interfaces. The extra effort can therefore improve efficiency and safety of operation.

Acknowledgments. This work results from the ANIMS project, funded by the Eurocontrol Experimental Center via the CARE-INO initiative (Co-operative Actions of R\&D in EUROCONTROL - Innovation), and conducted in collaboration with Intactile Design.

\section{References}

1. Athènes, S., Chatty, S., Bustico, A.: Human factors in ATC alarms and notifications design: experimental evaluation. In: Proc. ATM'2000 R\&D seminar (2000)

2. Bartram, L., Ware, C., Calvert, T.: Moving icons: Detection and distraction. In: Proc. Interact'01,IFIP. IOS Press, Amsterdam (2001)

3. Bertin, J.: Sémiologie graphique. Les diagrammes - Les réseaux - Les plans, «Les Réimpressions », 4ème éd (1ère éd.: 1967), Editions de l'EHESS (2005)

4. Bezerianos, A., Dragicevic, P., Balakrishnan, R.: Mnemonic Rendering: An Image-Based Approach for Exposing Hidden Changes in Dynamic Displays. In: Proc. UIST2006 (2006) (to appear)

5. Chang, B.-W., Ungar, D.: Animation: From Cartoon to User Interface. In: Proc. UIST'93, pp. 45-55 (1993)

6. Cockburn, A., Brewster, S.A.: Multimodal feedback for the acquisition of small targets. Ergonomics 48(9), 1129-1150 (2005)

7. Crease, M.G., Brewster, S.A.: Making Progress With Sounds - The Design and Evaluation Of An Audio Progress Bar. In: Proc. ICAD'98 (1998)

8. Endsley, M.R.: Toward a theory of situation awareness. Human Factors 37(1), 32-64 (1995)

9. Gaver, W.: The SonicFinder: an interface that uses auditory icons. Human Computer Interaction 4(1), 67-94 (1989)

10. Gaver, W.W., Smith, R.B., O'Shea, T.: Effective sounds in complex systems: the ARKOLA simulation. In: Proc. CHI '91, pp. 85-90. ACM Press, New York (1991)

11. Gonzalez, C.: Does animation in user interfaces improve decision making? In: Proc. CHI '96, pp. 27-34. ACM Press, New York (1996)

12. Igarashi, T., Mackinlay, J.D., Chang, B.-W., Zellweger, P.T.: Fluid Visualization of Spreadsheet Structures. In: Proc. Visual Languages'99, pp. 118-125 (1999) 
13. Jacko, J.A., Scott, I.U., Sainfort, F., Barnard, L., Edwards, P.J., Emery, V.K., Kongnakorn, T., Moloney, K.P., Zorich, B.S.: Older adults and visual impairment: what do exposure times and accuracy tell us about performance gains associated with multimodal feedback? In: Proc. CHI '03, pp. 33-40. ACM Press, New York (2003)

14. McCrickard, D.S.: Maintaining Information awareness with Irwin. In: Proc. ACM CSCW, pp. 314-323. ACM Press, New York (2002)

15. Mertz, C., Chatty, S., Vinot, J.-L.: Pushing the limits of ATC user interface design beyond S\&M interaction: the DigiStrips Experience. In: Proc. ATM'2000 R\&D seminar (2000)

16. Mynatt, E.D., Back, M., Want, R., Baer, M., Ellis, J.B.: Designing audio aura. In: Proc. CHI'98, pp. 566-573. ACM Press/Addison-Wesley (1998)

17. Patterson, R.D., Edworthy, J., Shailer, M.J., et al.: Alarm sounds for medical equipment in intensive care areas and operating theatres, Report No. AC598. Institute of Sound and Vibration Research, Southampton, UK (1986)

18. Robertson, G.G., Mackinlay, J.D., Card, S.K.: Cone Trees: animated 3D visualizations of hierarchical information. In: Proc. CHI '91, pp. 189-194. ACM Press, New York (1991)

19. Rensink, R.A., O'Regan, J.K., Clark, J.J.: To see or not to see: the need for attention to perceive changes in scenes. Psychological Science 8(8), 368-373 (1997)

20. Vitense, H.S., Jacko, J.A., Emery, V.K.: Multimodal feedback: An assessment of performance and mental workload. Ergonomics 46(1-3), 58-87 (2003)

21. Ware, C., Neufeld, E., Bartram, L.: Visualizing Causal Relations. In: Proc. IEEE InfoViz'99, pp. 39-42. IEEE Computer Society Press, Los Alamitos (1999)

22. Ware, C.: Information visualization: perception for design. Morgan Kaufmann, San Francisco (2000)

23. Wickens, C.D.: Engineering psychology and human performance, Columbus, Merrill, CE (1984)

24. Woods, D.D., O’Brien, J., Hanes, L.F.: Human Factors challenges in process control, the case of nuclear power plants. In: Salvendy, G. (ed.) Handbook of human factors/Ergnomics (1987)

25. Woods, D.D: The alarm problem and directed attention in dynamic fault management. Ergonomics (38), 2371-2393 (1995) 